\title{
Coherent photo-nuclear production of vector mesons at mid-rapidities in $\mathrm{Pb}-\mathrm{Pb}$ collisions
}

\author{
Guillermo Contreras* \\ (on behalf of the ALICE Collaboration) \\ Faculty of Nuclear Sciences and Physical Engineering, Czech Technical University in Prague, \\ Prague, Czech Republic \\ E-mail: jgcn@mail.cern.ch
}

\begin{abstract}
Recent measurements of coherent photo-nuclear production of vector mesons at mid-rapidity, performed by the ALICE Collaboration at the LHC in ultra-peripheral collisions of lead ions, are briefly summarised.
\end{abstract}

XXIV International Workshop on Deep-Inelastic Scattering and Related Subjects

11-15 April, 2016

DESY Hamburg, Germany

${ }^{*}$ Speaker. 


\section{The $\mathrm{L} \gamma \mathrm{HC}$}

The electromagnetic field of ultra relativistic charged particles can be interpreted as a flux of quasi-real photons. Their virtuality is limited, through the uncertainty principle, by the size of the emitting particle. When the source is a lead nucleus the virtuality is smaller than $\approx 30 \mathrm{MeV}$ for a heavy nucleus like gold or lead. Their energy spans from very soft photons up to a maximum energy given by the boost of the source in the laboratory frame. This means, that the LHC is also a photon-hadron and photon-photon collider, which is capable of producing these collisions in a wide range of centre-of-mass energies. As the intensity of the photon flux depends quadratically on the charge of the particle, lead ions produce abundant $\gamma \mathrm{Pb}$ interactions in $\mathrm{Pb}-\mathrm{Pb}$ collisions.

The challenge is to separate photon-induced processes from all other types of collisions at the LHC. One option is to select a special type of interactions where there are large rapidity gaps, because $\mathrm{Pb}-\mathrm{Pb}$ hadronic collisions tend to produce particles at all rapidities. The physics picture is that the incoming particles interact at impact parameters larger than the sum of their radii, thus strongly suppressing hadronic collisions, such that only photon-mediated interactions are possible. Because of this impact-parameter condition, these events are called ultra-peripheral collisions (UPC).

Among UPC there is a special set of processes with a very clean experimental signature and which probe directly the gluon distribution of the hadronic target. In these processes, the photon interacts with the target, without breaking it, to produce a vector meson. When the target is the full nucleus the process is called coherent, when the target is a nucleon inside the nucleus the process is incoherent.

The experimental signature is quite striking, since only the daughter tracks of the decay of the vector meson are seen in the main detector. The total transverse momentum is very small, less than some $50 \mathrm{MeV}$ (plus effects of the detector resolution) for coherent and around $500 \mathrm{MeV}$ for incoherent production. A brief overview of the main results from the ALICE Collaboration for vector mesons measured at mid-rapidity is presented below. A more complete and detailed recent review of this field at the LHC is given in [1].

\section{The ALICE detector}

The ALICE detector consists of two main sections, a central barrel(CB) and a muon spectrometer in the forward direction. A full description of ALICE is given in [2], while its performance is reported in [3].

The $\mathrm{CB}$ is located within a large solenoid magnet providing a uniform magnetic field of 0.5 T. The pseudorapidity coverage for tracks traversing the full set of central detectors is $|\eta|<0.9$. The CB detectors used in the measurements described below are the inner tracker system (ITS), the time projection chamber (TPC) and the time-of-flight detector (TOF). The ITS has six layers of silicon detectors. From the inside to the outside there are two layers of silicon pixels (SPD), two layers of silicon drift and two layers of silicon strip detectors. The TPC is the main tracking device of ALICE and is used for tracking and particle identification (PID) using ionisation energy loss. The TPC is a cylindrical gaseous detector with a diameter of $500 \mathrm{~cm}$ and a length of $510 \mathrm{~cm}$ which can provide up to 159 points for the track and PID measurements. The TOF detector, which 

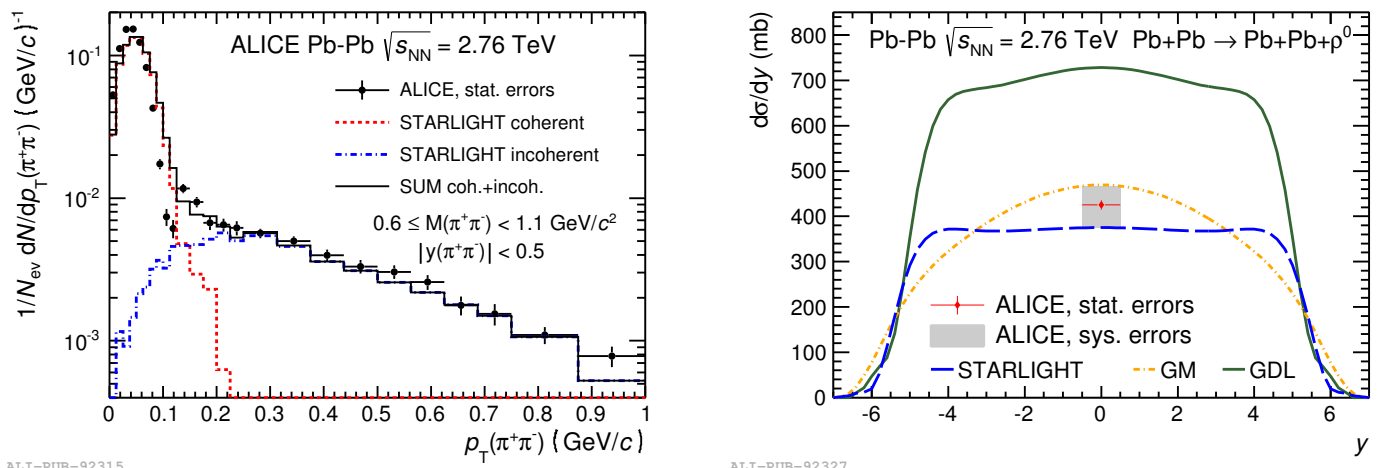

Figure 1: Distribution of transverse momentum for $\rho^{0}$ candidates in UPC (left panel) and measured cross section for coherent $\rho^{0}$ production in $\mathrm{Pb}-\mathrm{Pb}$ collisions at $\sqrt{s_{\mathrm{NN}}}=2.76 \mathrm{TeV}$ (right panel). Details are in the text. Figures from [4].

surrounds the TPC, is composed of multi-gap resistive plate chambers which provide an intrinsic time resolution of approximately $80 \mathrm{ps}$. In the analyses presented here it is used to trigger UPC events at mid-rapidity.

Other detectors relevant for the analyses reported below are the V0 and the zero degree calorimeters (ZDC). The V0 consists of two arrays of 32 scintillator tiles each, covering the ranges $2.8<\eta<5.1$ (V0A) and $-3.7<\eta<-1.7$ (V0C). The time resolution of each array is $\leq 1 \mathrm{~ns}$ and they form part of the level-0 trigger. They are also used to veto activity in the detector beyond the daughter particles of the vector meson. There are two ZDCs located at $\pm 116 \mathrm{~m}$ from the interaction point. They can detect neutrons produced in the very forward regions. In the analyses presented here the neutron ZDCs are used to veto hadronic interactions, as well as to signal incoherent interactions, which are accompanied by one or a few neutrons at very large rapidities.

\section{Results from $\mathbf{P b}-\mathrm{Pb}$ collisions at mid rapidity}

ALICE has measured the following decay channels at mid-rapidity: $\rho \rightarrow \pi^{+} \pi^{-}, \mathrm{J} / \psi \rightarrow l^{+} l^{-}$, where $l$ stands for either an electron or a muon, and $\psi(2 \mathrm{~S}) \rightarrow l^{+} l^{-}$and $\psi(2 \mathrm{~S}) \rightarrow \mathrm{J} / \psi \pi^{+} \pi^{-}$. As the transverse momentum of the vector meson is very small in the laboratory system, the daughter particles from two-body decays are produced back-to-back in azimuth and carry also a small transverse momentum. For the corresponding momentum ranges the PID capabilities of the TPC are able to separate electrons from muons and/or pions. Furthermore, for the case of the $\psi(2 \mathrm{~S}) \rightarrow \mathrm{J} / \psi \pi^{+} \pi^{-}$ decay, the pions have smaller momentum than the leptons from the $\mathrm{J} / \psi$ decay, which allows to distinguish them using kinematics. In summary, it is possible to identify effectively the decay products. In all channels the background from same-sign tracks under the mass peaks is either negligible or very small.

\subsection{Coherent production of $\rho^{0}$}

The coherent production of $\rho^{0}$ vector meson was measured with 2010 data from $\mathrm{Pb}-\mathrm{Pb}$ UPC at a centre-of-mass energy per nucleon pair of $\sqrt{s_{\mathrm{NN}}}=2.76 \mathrm{TeV}$ [4]. Two triggers were used. One 

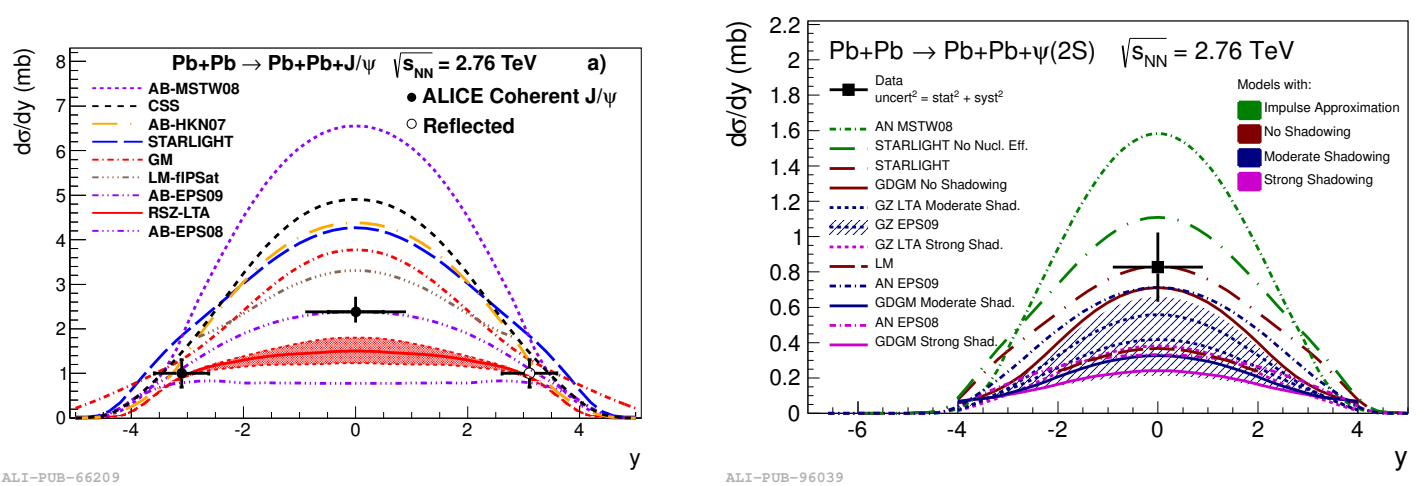

Figure 2: Measured cross section for coherent $\mathrm{J} / \psi$ (left panel) and $\psi(2 \mathrm{~S})$ (right panel) production in $\mathrm{Pb}-\mathrm{Pb}$ collisions at $\sqrt{s_{\mathrm{NN}}}=2.76 \mathrm{TeV}$. Details are in the text. Figures from [9] and [10].

when the instantaneous luminosity was low required at least two hits in the TOF detector. The second, active when the instantaneous luminosity increased, required in addition two hits in the outer layer of the SPD and no activity in any of the V0 arrays.

Two well reconstructed tracks, identified as pions using the TPC, and no other activity in the $\mathrm{CB}$, nor in the $\mathrm{V} 0$ detectors was required in the offline analysis. The four-momenta of the two tracks were used to compute the kinematics of the $\rho^{0}$ candidates. These were selected with a rapidity $|y|<0.5$. The distribution of transverse momentum $\left(p_{T}\right)$ of the pion pair was used to separate a coherent enriched sample by selecting candidates around the $\rho^{0}$ mass to have less than $150 \mathrm{MeV} / c$ of $p_{T}$ (see left panel of Fig. 1). After correcting it from acceptance and efficiency of the detector using specialised MC simulations, the mass distribution was fitted to a Söding distribution and the Breit-Wigner part was used to estimate the number of $\rho^{0}$ candidates.

The measured cross section is shown in the right panel of Fig. 1, where it is compared to the predictions of three models: STARLIGHT [5], GM [6] and GDL [7]. The best description of data is achieved by the STARLIGHT and GM models. STARLIGHT is based on a fit to $\gamma p$ data, the vector-dominance model and a classical Glauber calculation to take into account nuclear effects. GM is based on the colour dipole model with a CGC-inspired prescription for saturation. The GDL model is based on the leading-twist approximation, fitted to HERA diffraction data. Both models use a standard Glauber calculation to go from $\gamma \mathrm{p}$ to $\gamma \mathrm{Pb}$. The authors of the GDL model have found that the inclusion of inelastic shadowing helps to improve the agreement of their model with data [8].

\subsection{Coherent production of $\mathrm{J} / \psi$}

The coherent and incoherent production of $\mathrm{J} / \psi$ at mid rapidity was measured with 2011 data from $\mathrm{Pb}-\mathrm{Pb} \mathrm{UPC}$ at a centre-of-mass energy per nucleon pair of $\sqrt{s_{\mathrm{NN}}}=2.76 \mathrm{TeV}$ [9]. The trigger was similar to the second one used for the $\rho^{0}$ analysis, with the addition that the TOF signals had to be back-to-back in azimuth. The selection of events and requirements for tracks were also similar. The PID capabilities of the TPC were used to separete the decays of the $\mathrm{J} / \psi$ to $e^{+} e^{-}$from those to $\mu^{+} \mu^{-}$. In the first (second) case, a coherent enriched sample was created selecting candidates with $p_{T}<300(200) \mathrm{MeV}$. 
The measured cross section is shown in the left panel of Fig. 2. In the same figure the measurement at forward rapidities by ALICE reported in [11] is also shown. The measurement is compared to the three models previously mentioned and in addition to the AB [12], LM [13] and CSS [14] models. LM and CSS use a dipole model, LM with a saturation prescription and CSS based directly on the unintegrated gluon distribution. The AB model used the LO pQCD expression, with the gluon distribution modified by a series of different shadowing factors to assess the sensitivity of the measurement to current nuclear PDF parameterisations. The best description of data is obtained with EPS09.

\subsection{Coherent production of $\psi(2 \mathrm{~S})$}

The coherent production of $\psi(2 \mathrm{~S})$ at mid rapidity was measured with 2011 data from $\mathrm{Pb}-\mathrm{Pb}$ UPC at a centre-of-mass energy per nucleon pair of $\sqrt{s_{\mathrm{NN}}}=2.76 \mathrm{TeV}$ [10]. The trigger was the same as used for the $\mathrm{J} / \psi$ analysis. For the decay into lepton pairs the selection was also the same as for the $\mathrm{J} / \psi$ analysis. For the decay channels $\psi(2 \mathrm{~S}) \rightarrow \mathrm{J} / \psi \pi^{+} \pi^{-}$the track requirements were slightly relaxed in order to optimise the selection.

The different measured cross sections were combined using the statistical and uncorrelated uncertainties as a weight. The resulting measurement is shown in the right panel of Fig. 2. The cross section is compared to the same set of models as for the $\mathrm{J} / \psi$. The GM model was updated to the GDGM model [15] using the by then published measurement of coherent $\mathrm{J} / \psi$ production. Again the EPS09 parameterisation describes best the data.

\section{Perspectives for the LHC Run2}

The Run2 at the LHC has already produced $\mathrm{Pb}-\mathrm{Pb}$ collisions and a new data taking period is expected to take place in the next years. These collisions are now at $\sqrt{s_{\mathrm{NN}}}=5.02 \mathrm{TeV}$, namely twice as what was achieved in Run 1 . The measurements at mid rapidity will be sensitive to larger $\gamma \mathrm{Pb}$ energies, correspondingly, smaller $x$ values of the nuclear gluon distribution.

Besides the improvements expected from the increased energy, ALICE has incorporated a new set of detectors, $\mathrm{AD}$, consisting of two arrays of scintillators placed at both sides of the nominal interaction point and covering the pseudorapidity intervals $-6.9<\eta<-4.9$ and $4.9<\eta<4.3$. This enlarged coverage will improved the capabilities of ALICE to impose a veto on extra activity in the events.

In the data taking period of 2015, ALICE accumulated already 6 times more integrated luminosity than what is available from 2011 for UPC analyses. Furthermore, the increase of LHC energy implies a more intense flux of photons at mid rapidity, so the expected number of events is even larger than the increase expected just by the luminosity. This larger data sample, will allow ALICE to improve the already existing measurements and attempt new measurements that were not possible before.

An exciting set of new measurements from ALICE in the field of UPC are coming in the near future.

Aknowledgements This work was supported by grant LK11209 of MŠMT ČR. 


\section{References}

[1] J. G. Contreras and J. D. Tapia Takaki, Ultra-peripheral heavy-ion collisions at the LHC, Int. J. Mod. Phys. A30 (2015) 1542012.

[2] ALICE collaboration, K. Aamodt et al., The ALICE experiment at the CERN LHC, JINST 3 (2008) S08002.

[3] ALICE collaboration, B. B. Abelev et al., Performance of the ALICE Experiment at the CERN LHC, Int. J. Mod. Phys. A29 (2014) 1430044, [1402.4476].

[4] ALICE collaboration, J. Adam et al., Coherent $\rho^{0}$ photoproduction in ultra-peripheral $\mathrm{Pb}-\mathrm{Pb}$ collisions at $\sqrt{s_{\mathrm{NN}}}=2.76 \mathrm{TeV}$, JHEP 09 (2015) 095, [1503.09177].

[5] S. Klein and J. Nystrand, Exclusive vector meson production in relativistic heavy ion collisions, Phys. Rev. C60 (1999) 014903, [hep-ph/9902259].

[6] V. P. Goncalves and M. V. T. Machado, Vector Meson Production in Coherent Hadronic Interactions: An update on predictions for RHIC and LHC, Phys. Rev. C84 (2011) 011902, [1106.3036].

[7] V. Rebyakova, M. Strikman and M. Zhalov, Coherent $\rho$ and J/ $\psi$ photoproduction in ultraperipheral processes with electromagnetic dissociation of heavy ions at RHIC and LHC, Phys. Lett. B710 (2012) 647-653, [1109.0737].

[8] L. Frankfurt, V. Guzey, M. Strikman and M. Zhalov, Nuclear shadowing in photoproduction of $\rho$ mesons in ultraperipheral nucleus collisions at RHIC and the LHC, Phys. Lett. B752 (2016) 51-58, [1506.07150].

[9] ALICE collaboration, E. Abbas et al., Charmonium and $e^{+} e^{-}$pair photoproduction at mid-rapidity in ultra-peripheral Pb-Pb collisions at $\sqrt{s_{\mathrm{NN}}}=2.76$ TeV, Eur. Phys. J. C73 (2013) 2617, [1305. 1467$]$.

[10] ALICE collaboration, J. Adam et al., Coherent $\psi(2 S)$ photo-production in ultra-peripheral $\mathrm{Pb} \mathrm{Pb}$ collisions at $\sqrt{s}_{\mathrm{NN}}=2.76$ TeV, Phys. Lett. B751 (2015) 358-370, [1508.05076].

[11] ALICE collaboration, B. Abelev et al., Coherent $\mathrm{J} / \psi$ photoproduction in ultra-peripheral Pb-Pb collisions at $\sqrt{s_{N N}}=2.76$ TeV, Phys. Lett. B718 (2013) 1273-1283, [1209.3715].

[12] A. Adeluyi and C. A. Bertulani, Constraining Gluon Shadowing Using Photoproduction in Ultraperipheral pA and AA Collisions, Phys. Rev. C85 (2012) 044904, [1201.0146].

[13] T. Lappi and H. Mantysaari, J/ $\psi$ production in ultraperipheral $\mathrm{Pb}+\mathrm{Pb}$ and $\mathrm{p}+\mathrm{Pb}$ collisions at energies available at the CERN Large Hadron Collider, Phys. Rev. C87 (2013) 032201, [1301.4095].

[14] A. Cisek, W. Schafer and A. Szczurek, Exclusive coherent production of heavy vector mesons in nucleus-nucleus collisions at LHC, Phys. Rev. C86 (2012) 014905, [1204.5381].

[15] M. B. G. Ducati, M. T. Griep and M. V. T. Machado, Diffractive photoproduction of radially excited psi(2S) mesons in photon-Pomeron reactions in PbPb collisions at the CERN LHC, Phys. Rev. $\mathbf{C 8 8}$ (2013) 014910, [1305.2407]. 